Fernández-García, C., Salas-Moya, C., Mena, S., AzofeifaSolano, J. C., \& Alvarado, J. J. (2021). Diversidad de los hábitats submareales de la Península de Santa Elena e Islas Murciélago, Pacífico Norte, Costa Rica. Revista de Biología Tropical, 69(Suppl. 2), S160-S179. https://doi. org/10.15517/rbt.v69iS2.48774

\title{
Diversidad de los hábitats submareales de la Península de Santa Elena e Islas Murciélago, Pacífico Norte, Costa Rica
}

\author{
Cindy Fernández-García ${ }^{1,2,3}$; (D) https://orcid.org/0000-0003-2808-4093 \\ Carolina Salas-Moya ${ }^{1}$; (D) https://orcid.org/0000-0002-3781-8127 \\ Sebastián Mena1; (D) https://orcid.org/0000-0002-1403-5533 \\ Juan Carlos Azofeifa-Solano2; (D) https://orcid.org/0000-0002-2105-1056 \\ Juan José Alvarado ${ }^{1,2,3}$; (D) https://orcid.org/0000-0002-2620-9115
}

1. Escuela de Biología, Universidad de Costa Rica, San Pedro, 2060-1000 San José, Costa Rica;

cindy.fernandezgarcia@ucr.ac.cr; caro.salasm@gmail.com; sebas.menago@gmail.com; juan.alvarado@ucr.ac.cr

2. Centro de Investigación en Ciencias del Mar y Limnología (CIMAR), Universidad de Costa Rica, San Pedro, 20601000 San José, Costa Rica; juan.azofeifa@ucr.ac.cr

3. Centro de Investigación en Biodiversidad y Ecología Tropical (CIBET), Universidad de Costa Rica, San Pedro, 20601000 San José, Costa Rica.

$$
\text { Recibido 30-I-2021. Revisado 07-V-2021. Aceptado 30-VII-2021. }
$$

\author{
ABSTRACT \\ Subtidal habitats diversity of Santa Elena Peninsula and Murciélago Islands, \\ North Pacific, Costa Rica
}

Introduction: Worldwide, coastal habitats are experiencing increasing pressure from pollution, coastal development, fisheries, and climate change. Identifying and recording coastal biodiversity is essential to assess ecosystem health, changes and the extent of biodiversity loss. Coastal tropical habitats such as coral reefs and seagrass beds have been the research focus for scientists during the last decades; however, other ecosystems have been neglected, such as macroalgae forests, subtidal (20-30 m deep) sedimentary bottoms, and rocky reefs.

Objective: Our study reports the marine biodiversity associated with rocky reefs, coral reefs, Sargassum forests and sedimentary subtidal bottoms (20-30 m deep), in a tropical seasonal upwelling area (Santa Elena Peninsula, Santa Elena Bay, and Murciélago Islands, Costa Rica).

Methods: During the 'Santa Elena Expedition' (April 21-May 2 2018), a total of 28 sites were visited in order to record the biodiversity across four different habitats (rocky and coral reefs, Sargassum forests, subtidal sedimentary bottoms), using SCUBA and both systematic surveys and visual assessments; in some sedimentary bottoms a 20-minute dredge tows were done.

Results: A total of 254 taxa were identified, being bony fishes the most diverse group ( 91 species), followed by gastropods (25 spp.), red algae (21 spp.) and anthozoans (19 spp.). We report four new records for the Pacific mainland of Costa Rica, including the subclass of tube-dwelling sea anemone (Ceriantharia).

Conclusions: Our results show that Santa Elena Peninsula, Santa Elena Bay, and Murciélago Islands harbor uncommon and unstudied habitats, such as Sargassum forests, sedimentary bottoms, reefs constructed by the stony coral Pavona gigantea -which is very unusual- and rocky reef communities dominated by calcareous colonies of polychaetes (Salmacina tribranchiata).

Key words: coral reefs; Eastern Tropical Pacific; Sargassum forest; sandy bottoms; Área de Conservación Guanacaste. 
Los ecosistemas marino-costeros y su biodiversidad están experimentando una presión cada vez mayor en todo el mundo debido a la contaminación, a la mala planificación de infraestructura, a la sobrepesca y al cambio climático (He \& Silliman, 2019). Conocer la biodiversidad marina es esencial para evaluar el estado, los cambios y el alcance de la pérdida en estos ecosistemas (Costello, Michener, Gahegan, Zhang, \& Bourne, 2013; Worm et al., 2006). Los hábitats marino-costeros tropicales, como los arrecifes de coral, los manglares, zonas intermareales y los lechos de pastos marinos, han sido el foco de investigación en las últimas décadas. Sin embargo, en muchos sitios el estudio de otros ecosistemas, como los bosques submarinos de algas o los fondos submareales de sustratos blandos ha sido menor (Gouvêa et al., 2020; Mecho, Easton, Sellanes, Gorny, \& Mah, 2019).

En el Pacífico costarricense, los ambientes marinos más estudiados han sido principalmente los arrecifes rocosos, los arrecifes y comunidades coralinas, las zonas rocosas intermareales, las playas y los manglares (Cortés, 2016). En Costa Rica, los estudios de diversidad se han concentrado en los ambientes más someros, desde las zonas intermareales hasta los $10 \mathrm{~m}$ de profundidad, posiblemente relacionado con las facilidades en el muestreo y con especies de importancia comercial, y por lo tanto son sitios que han tenido mejores medidas de conservación en el país (Alvarado, Beita-Jimenez, Mena, FernándezGarcía, Cortés, Sánchez-Noguera, Jimenez \& Guzmán-Mora, 2018; Alvarado, Beita, Mena, Fernández-García, \& Guzman-Mora, 2015; Alvarado, Beita, Mena, Fernández-García, Guzman-Mora \& Cortés, 2016; Alvarado, Herrera, Corrales, Asch, \& Paaby, 2011; Alvarado, Sánchez-Noguera, Arias-Godínez, Araya, Fernández-García \& Guzmán, 2020; SamperVillareal, Bourg, Sibaja-Cordero, \& Cortés, 2014; Sibaja-Cordero \& Cortés, 2009; SibajaCordero \& Vargas-Zamora, 2006). También se han realizado investigaciones en ambientes que van de los 10 a los $40 \mathrm{~m}$ utilizando buceo autónomo, no obstante, están más relacionados con estudios taxonómicos en grupos como los octocorales (Breedy \& Guzmán, 2003).

Recientemente, varios trabajos han recopilado la diversidad marina del Área de Conservación Guanacaste (ACG), enfocándose en la revisión de colecciones y literatura científica, así como del Proyecto BioMar-ACG (ChacónMonge, Azofeifa-Solano, Alvarado, \& Cortés, 2021; Cortés, 2017; Cortés \& Joyce, 2020; Vargas \& Cortés, 2019). No obstante, hay muy pocas investigaciones que estudien la diversidad de los bosques de Sargassum (ver Suárez, Riosmena-Rodríguez \& Cortés, 2021) y de zonas arenosas a profundidades mayores de los $20 \mathrm{~m}$, entre otros ambientes. A pesar de que estos ecosistemas resguardan una diversidad muy específica, en Costa Rica se han investigado poco (Cortés, Samper-Villarreal, \& Bernecker, 2014). Por lo tanto, el objetivo de esta investigación es caracterizar la biodiversidad de ambientes submareales en la península de Santa Elena y las Islas Murciélago, en el Pacífico norte de Costa Rica.

\section{MATERIALES Y MÉTODOS}

Sitio de estudio: Los ambientes costeros del ACG incluyen accidentes geográficos característicos, tales como playas, costas rocosas, bahías e islotes en la Península de Santa Elena, la Bahía Santa Elena y las Islas Murciélago (Fig. 1). Actualmente, están bajo alguna categoría de protección $430 \mathrm{~km}^{2}$ y 150 km de línea de costa del Parque Nacional Santa Rosa, así como $7.28 \mathrm{~km}^{2}$ del Área Marina de Manejo Bahía Santa Elena. Esta zona presenta una estacionalidad marcada debido al afloramiento costero, que ocurre de diciembre y marzo, meses en los cuales se reportan bajas temperaturas y un aumento en la concentración de nutrientes en la columna de agua (Alvarado et al., 2018; Legeckis, 1988; Lizano \& Alfaro, 2014; McCreary, Lee, \& Enfield, 1989; Rodríguez, Alfaro \& Cortés, 2021).

La Península de Santa Elena está compuesta por una secuencia de basaltos alcalinos, radiolarita y brechas polimícticas que datan del Jurásico-Cretácico (Denyer, Cortés, 


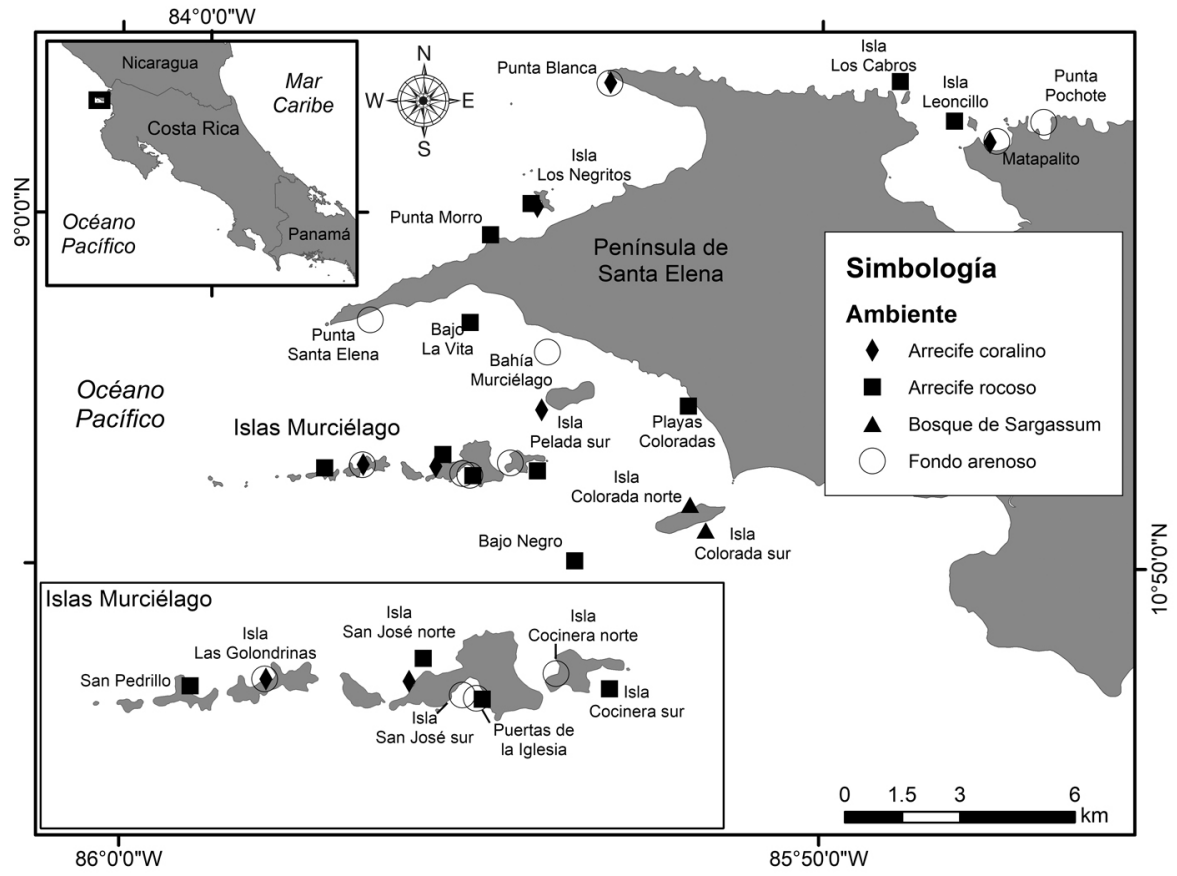

Fig. 1. Sitios de muestreo de distintos hábitats submareales durante la expedición Santa Elena Waitt Foundation-MarViva (2018), Pacífico Norte de Costa Rica.

Fig. 1. Sampling sites at different subtidal habitats during the Santa Elena expedition Waitt Foundation-MarViva (2018), North Pacific of Costa Rica.

\& Cárdenes, 2005; Denyer \& Gazel, 2009). La Bahía de Santa Elena es relativamente somera, con las mayores profundidades entre 30 y 35 $\mathrm{m}$ en su zona externa; está rodeada por playas de arena hacia las zonas externas, y planicies de limo y arena hacia la zona interna cerca del Estero Grande, con fondos submareales dominados por arena y rocas coralinas en algunas zonas (BIOMARCC-SINAC-GIZ, 2012). Por su parte, las Islas Murciélago, compuestas por cinco islas principales y al menos 10 rocas e islotes, se ubican a $5 \mathrm{~km}$ al sur de la península, y están conformadas por basaltos columnares, en almohadilla y masivos que datan del Cretácico (Denyer et al., 2005; Hauff et al., 2000).

Recolecta de datos: Del 21 de abril al 02 de mayo del 2018 se visitaron un total de 28 sitios para analizar los hábitats costeros de la Península de Santa Elena, Bahía Santa Elena e Islas Murciélago durante la Expedición Santa
Elena Waitt Foundation-MarViva, a bordo del yate Plan B (Fig. 1). Durante esta visita se realizó un listado de especies para cuatro tipos de hábitats submareales: arrecifes y comunidades coralinas, arrecifes rocosos, bosques del alga parda Sargassum y fondos de sedimentos.

En los arrecifes y las comunidades coralinas, los arrecifes rocosos y los bosques de Sargazo se realizaron censos visuales para determinar la diversidad de peces e invertebrados móviles y la composición del sustrato (invertebrados sésiles y algas) siguiendo la metodología de Alvarado et al. (2018) y Alvarado et al. (en prensa). Para el caso de fondos arenosos, se hicieron buceos a una profundidad inicial máxima de $35 \mathrm{~m}$, en los cuales se realizaron transectos perpendiculares de $10 \mathrm{~m}$ a la costa siguiendo un rumbo, hasta llegar a los $5 \mathrm{~m}$ de profundidad. En estos transectos se anotaron las especies de vertebrados observadas y se recolectaron macroinvertebrados 
móviles y sésiles, además de algas. Adicionalmente, se realizaron observaciones mediante buceos errantes y se recolectaron organismos manualmente durante los buceos con SCUBA. En algunos fondos blandos se utilizó una draga metálica $(50 \mathrm{~cm} \times 25 \mathrm{~cm})$ arrastrada sobre el fondo durante 20 minutos, entre 15 y $30 \mathrm{~m}$ de profundidad. Los organismos de esta expedición científica fueron depositados en las colecciones del Herbario Dr. Luis A. Fournier Origgi (USJ) y el Museo de Zoología (MZUCR) de la Universidad de Costa Rica.

\section{RESULTADOS}

En total se observaron 254 especies de organismos marinos, distribuidas en 192 géneros, 153 familias, 69 órdenes, 17 clases y 10 filos (Tabla 1). Entre estas se encuentran cuatro reportes nuevos para el país, incluyendo una especie de alga (Scinaia spp., que no coincide con ninguna de las especies del género conocidas para el Pacífico Tropical Oriental), dos géneros de algas (Gaylliela y Siphonocladus) y la subclase de anémonas tubícolas Ceriantharia (orden Spirularia, familia Cerianthidae). El grupo con mayor riqueza de especies fueron los peces óseos (90), seguidos de Gastropoda con (25 spp.), las algas rojas del filo Rhodophyta (20 spp.) y Anthozoa (19 spp.) (Tabla 1, Fig. 2).

De los cuatro hábitats principales visitados durante la expedición (Fig. 1), los arrecifes rocosos tuvieron la mayor riqueza de especies con 175 en total (Tabla 1, Fig. 3), correspondientes a 78 especies de peces, 25 de algas (pardas, verdes y rojas), y 24 especies de moluscos, entre otras. Los arrecifes de coral fueron segundos en términos de riqueza con 130 especies en total, incluyendo 52 de peces óseos y batoideos, 24 de algas, y 14 especies de cnidarios (corales escleractinios y corales

TABLA 1

Taxonomía de las especies encontradas por ambiente durante la Expedición Santa Elena Waitt-MarViva, Pacífico norte de Costa Rica, 2018. AR: Arrecife rocoso; AC: Arrecife de Coral; BS: Bosque de Sargassum; FA: Fondo arenoso

TABLE 1

Taxonomy of the species found by environment during the Waitt-MarViva Santa Elena Expedition, North Pacific of Costa Rica, 2018. AR: Rocky reef; AC: Coral Reef; BS: Sargassum forest; FA: Sandy bottom

\begin{tabular}{|c|c|c|c|c|c|c|c|c|}
\hline Taxa & $\mathrm{AR}$ & $\mathrm{AC}$ & $\mathrm{BS}$ & FA & Taxa & $\mathrm{AR}$ & $\mathrm{AC} \mathrm{BS}$ & FA \\
\hline Filo Chlorophyta & & & & & Orden Ulvales & & & \\
\hline Clase Ulvophyceae & & & & & Familia Ulvaceae & & & \\
\hline Orden Bryopsidales & & & & & Ulva cf. lactuca & $\mathrm{X}$ & $\mathrm{X}$ & $\mathrm{X}$ \\
\hline Familia Bryopsidaceae & & & & & Ulva cf. lingulata & & $\mathrm{X}$ & \\
\hline Bryopsis pennata & $\mathrm{X}$ & $\mathrm{X}$ & & & Filo Rhodophyta & & & \\
\hline Familia Caulerpaceae & & & & & Clase Florideophyceae & & & \\
\hline Caulerpa chemnitzia & $\mathrm{X}$ & $\mathrm{X}$ & $\mathrm{X}$ & & Orden Bonnemaisoniales & & & \\
\hline Caulerpa sertularioides & & & $\mathrm{X}$ & $\mathrm{X}$ & Familia Bonnemaisoniaceae & & & \\
\hline Familia Codiaceae & & & & & Asparagopsis taxiformis & $\mathrm{X}$ & $\mathrm{X}$ & \\
\hline Codium isabelae & $\mathrm{X}$ & & $\mathrm{X}$ & & Orden Ceramiales & & & \\
\hline Codium spp. & $\mathrm{X}$ & & $\mathrm{X}$ & & Familia Ceramiaceae & & & \\
\hline Familia Halimedaceae & & & & & Gayliella spp. & $\mathrm{X}$ & $\mathrm{X}$ & \\
\hline Halimeda discoidea & $\mathrm{X}$ & & $\mathrm{X}$ & & Ceramium spp. & $\mathrm{X}$ & $\mathrm{X}$ & $\mathrm{X}$ \\
\hline Familia Udoteaceae & & & & & Familia Delesseriaceae & & & \\
\hline Chlorodesmis caespitosa & $\mathrm{X}$ & & & & Hypoglossum spp. & & & $\mathrm{X}$ \\
\hline Orden Cladophorales & & & & & Familia Rhodomelaceae & & & \\
\hline Familia Siphonocladaceae & & & & & Chondria platyclada & & & $\mathrm{X}$ \\
\hline Siphonocladus spp. & & & & $\mathrm{X}$ & Orden Peyssonneliales & & & \\
\hline
\end{tabular}




\begin{tabular}{|c|c|c|c|c|c|c|c|c|c|}
\hline Taxa & $\mathrm{AR}$ & $\mathrm{AC}$ & BS & FA & Taxa & $\mathrm{AR}$ & $\mathrm{AC}$ & BS & FA \\
\hline Peyssonneliales spp. & $\mathrm{X}$ & $\mathrm{X}$ & & & Filo Cnidaria & & & & \\
\hline Orden Corallinales & & & & & Clase Anthozoa & & & & \\
\hline Familia Lithophyllaceae & & & & & Orden Actiniaria & & & & \\
\hline Amphiroa misakiensis & $\mathrm{X}$ & $\mathrm{X}$ & $\mathrm{X}$ & & Familia Hormathiidae & & & & \\
\hline Amphiroa sp. 1 & & $\mathrm{X}$ & & & Calliactis spp. & $\mathrm{X}$ & $\mathrm{X}$ & & \\
\hline Amphiroa sp. 2 & $\mathrm{X}$ & $\mathrm{X}$ & & & Orden Alcyonacea & & & & \\
\hline Lithophyllum spp. & $\mathrm{X}$ & $\mathrm{X}$ & & & Familia Clavulariidae & & & & \\
\hline Familia Lithothamniaceae & & & & & Carijoa riisei & & $\mathrm{X}$ & & \\
\hline Lithothamnion muelleri & $\mathrm{X}$ & & & & Familia Gorgoniidae & & & & \\
\hline Orden Gigartinales & & & & & Leptogorgia alba & $\mathrm{X}$ & $\mathrm{X}$ & $\mathrm{X}$ & \\
\hline Familia Cystocloniaceae & & & & & Orden Antipatharia & & & & \\
\hline Hypnea pannosa & $\mathrm{X}$ & $\mathrm{X}$ & & & Familia Antipathidae & & & & \\
\hline Hypnea spicifera & $\mathrm{X}$ & $\mathrm{X}$ & & & Antipathes spp. & $\mathrm{X}$ & & & \\
\hline Orden Gracilariales & & & & & Orden Pennatulacea & & & & \\
\hline Familia Gracilariaceae & & & & & Pennatulacea sp. 1 & & & & $\mathrm{X}$ \\
\hline Gracilaria sp. 1 & $\mathrm{X}$ & $\mathrm{X}$ & & $\mathrm{X}$ & Pennatulacea sp. 2 & & & & $\mathrm{X}$ \\
\hline Gracilaria sp. 2 & $\mathrm{X}$ & $\mathrm{X}$ & & & Familia Renillidae & & & & \\
\hline Gracilariopsis sp. & & & & $\mathrm{X}$ & Renilla spp. & & & & $\mathrm{X}$ \\
\hline Orden Halymeniales & & & & & Familia Virgulariidae & & & & \\
\hline Familia Halymeniaceae & & & & & Virgularia spp. & & & & $\mathrm{X}$ \\
\hline Halymenia spp. & & & & $\mathrm{X}$ & Orden Scleractinia & & & & \\
\hline Orden Nemaliales & & & & & Familia Agariciidae & & & & \\
\hline Familia Scinaiaceae & & & & & Pavona clavus & & $\mathrm{X}$ & & \\
\hline Scinaia spp. & & & & $\mathrm{X}$ & Pavona gigantea & $\mathrm{X}$ & $\mathrm{X}$ & $\mathrm{X}$ & \\
\hline Scinaia complanata & & & & $\mathrm{X}$ & Pavona varians & & $\mathrm{X}$ & & \\
\hline Familia Peyssonneliaceae & & & & & Pavona chiriquiensis & & $\mathrm{X}$ & & \\
\hline Peyssonnelia spp. & $\mathrm{X}$ & $\mathrm{X}$ & $\mathrm{X}$ & & Familia Dendrophylliidae & & & & \\
\hline Orden Rhodymeniales & & & & & Tubastraea coccinea & $\mathrm{X}$ & $\mathrm{X}$ & & \\
\hline Familia Rhodymeniaceae & & & & & Familia Pocilloporidae & & & & \\
\hline Botryocladia beaudettei & $\mathrm{X}$ & & & & Pocillopora spp. & $\mathrm{X}$ & $\mathrm{X}$ & $\mathrm{X}$ & \\
\hline Filo Ochrophyta & & & & & Familia Poritidae & & & & \\
\hline Clase Phaeophyceae & & & & & Porites panamensis & $\mathrm{X}$ & $\mathrm{X}$ & $\mathrm{X}$ & \\
\hline Orden Dictyotales & & & & & Porites lobata & $\mathrm{X}$ & $\mathrm{X}$ & $\mathrm{X}$ & \\
\hline Familia Dictyotaceae & & & & & Familia Psammocoridae & & & & \\
\hline Dictyota stolonifera & & $\mathrm{X}$ & $\mathrm{X}$ & $\mathrm{X}$ & Psammocora stellata & & $\mathrm{X}$ & & \\
\hline Dictyota humifusa & $\mathrm{X}$ & $\mathrm{X}$ & $\mathrm{X}$ & & Orden Spirularia & & & & \\
\hline Dictyota sp. nov. & $\mathrm{X}$ & $\mathrm{X}$ & & & Familia Cerianthidae & & & & \\
\hline Lobophora adpressa & & $\mathrm{X}$ & & & Cerianthidae sp. 1 & $\mathrm{X}$ & & & $\mathrm{X}$ \\
\hline Padina durvillei & $\mathrm{X}$ & $\mathrm{X}$ & $\mathrm{X}$ & & Orden Zoantharia & & & & \\
\hline Padina caulescens & $\mathrm{X}$ & $\mathrm{X}$ & $\mathrm{X}$ & & Familia Sphenopidae & & & & \\
\hline Orden Ectocarpales & & & & & Palythoa spp. & $\mathrm{X}$ & $\mathrm{X}$ & & \\
\hline Familia Scytosiphonaceae & & & & & Clase Hydrozoa & & & & \\
\hline Chnoospora implexa & $\mathrm{X}$ & $\mathrm{X}$ & & & Orden Anthoathecata & & & & \\
\hline Colpomenia sinuosa & & $\mathrm{X}$ & $\mathrm{X}$ & & Familia Pennariidae & & & & \\
\hline Rosenvingea intricata & & & & $\mathrm{X}$ & Pennaria spp. & & & & $\mathrm{X}$ \\
\hline Orden Fucales & & & & & Orden Leptothecata & & & & \\
\hline Familia Sargassaceae & & & & & Familia Aglaopheniidae & & & & \\
\hline Sargassum liebmannii & $\mathrm{X}$ & $\mathrm{X}$ & $\mathrm{X}$ & & Macrorhynchia philippina & $\mathrm{X}$ & $\mathrm{X}$ & $\mathrm{X}$ & \\
\hline
\end{tabular}




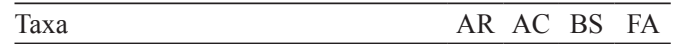

\section{Filo Phoronida}

Familia Phoronidae

Phoronopsis albomaculata

\section{Filo Annelida}

Clase Polychaeta

Orden Amphinomida

Familia Amphinomidae

Amphinomidae spp.

Orden Echiuroidea

Familia Echiuroidea

Echiuroidea spp.

Orden Phyllodocida

Familia Iphionidae

Iphione ovata

Familia Nereididae

Infraclase Canalipalpata

Familia Sabellariidae

Orden Sabellida

Familia Sabellidae

Sabellidae spp.

Familia Serpulidae

Serpulidae spp.

Filo Mollusca

Clase Bivalvia

Orden Arcida

Familia Arcidae

Arcidae spp.

Orden Cardiida

Familia Tellinidae

Tellina spp.

Orden Limida

Familia Limidae

Limaria pacifica

Orden Mytilida

Familia Mytilidae

Lithophaga sp.

Orden Nuculanida

Familia Nuculanidae

Nuculanidae spp.

Orden Ostreida

Familia Gryphaeidae

Hyotissa hyotis

X X

Familia Margaritidae

Pinctada mazatlanica

Familia Pinnidae

Atrina texta

Pinna rugosa

Orden Pectinida

X X

X
Taxa

AR AC BS FA

Familia Pectinidae

Pectinidae spp.

X Familia Spondylidae

Spondylus sp.

X

Orden Venerida

Familia Veneridae

Veneridae spp.

X Clase Cephalopoda

Orden Octopoda

Familia Octopodidae

X Octopus spp.

$\begin{array}{llll}X & X & X & X\end{array}$

Clase Gastropoda

Familia Plakobranchidae

X Plakobranchidae spp.

X Orden Aplysiida

Familia Aplysiidae

Stylocheilus striatus

Orden Littorinimorpha

Familia Bursidae

Bursidae spp.

Familia Cypraeidae

Eclogavena quadrimaculata

X X

X X

Familia Naticidae

Sinum spp.

Familia Ovulidae

Simnia avena

X X X

Familia Strombidae

Lobatus galeatus

Familia Tonnidae

X Malea ringens

Familia Vermetidae

Vermetidae sp.

X

X X

X X X

Orden Neogastropoda

Familia Columbellidae

X

Familia Fasciolariidae

Opeatostoma pseudodon

X X X

Triplofusus princeps

X

Familia Muricidae

X Hexaplex princeps

X

Murex spp.

X X

Familia Olividae

Oliva spp.

Olivella spp.

$\mathrm{X}$

X

Familia Terebridae

Terebridae sp.

X

X Familia Turbinellidae

Vasum spp.

X $\quad X$

Orden Nudibranchia 


\begin{tabular}{|c|c|c|c|c|c|c|c|c|c|}
\hline Taxa & $\mathrm{AR}$ & $\mathrm{AC}$ & BS & FA & Taxa & $\mathrm{AR}$ & $\mathrm{AC}$ & $\mathrm{BS}$ & FA \\
\hline Familia Chromodorididae & & & & & Portunidae sp. 2 & & & & $\mathrm{X}$ \\
\hline Doriprismatica sedna & $\mathrm{X}$ & $\mathrm{X}$ & $\mathrm{X}$ & & Familia Trapeziidae & & & & \\
\hline Felimare agassizii & $\mathrm{X}$ & & & & Trapezia bidentata & $\mathrm{X}$ & $\mathrm{X}$ & $\mathrm{X}$ & \\
\hline Familia Dorididae & & & & & Familia Xanthidae & & & & \\
\hline Conualevia alba & $\mathrm{X}$ & & & & Heteractaea lunata & $\mathrm{X}$ & & & \\
\hline Familia Facelinidae & & & & & Filo Echinodermata & & & & \\
\hline Favorinus spp. & $\mathrm{X}$ & & & & Clase Asteroidea & & & & \\
\hline Familia Fionidae & & & & & Orden Paxillosida & & & & \\
\hline Fiona pinnata & $\mathrm{X}$ & & & & Familia Astropectinidae & & & & \\
\hline Familia Flabellimidae & & & & & Astropecten spp. & & & & $\mathrm{X}$ \\
\hline Flabellina spp. & $\mathrm{X}$ & & & & Luidia spp. & & & & $\mathrm{X}$ \\
\hline Orden Pleurobranchida & & & & & Orden Valvatida & & & & \\
\hline Familia Pleurobranchidae & & & & & Familia Asteropseidae & & & & \\
\hline Berthellina ilisima & $\mathrm{X}$ & & & & Asteropsis carinifera & $\mathrm{X}$ & & & \\
\hline Filo Arthropoda & & & & & Familia Ophidiasteridae & & & & \\
\hline Clase Malacostraca & & & & & Pharia pyramidata & $\mathrm{X}$ & $\mathrm{X}$ & $\mathrm{X}$ & \\
\hline Orden Decapoda & & & & & Phataria unifascialis & $\mathrm{X}$ & $\mathrm{X}$ & $\mathrm{X}$ & \\
\hline Familia Alpheidae & & & & & Familia Oreasteridae & & & & \\
\hline Alpheus lottini & & $\mathrm{X}$ & & & Nidorellia armata & $\mathrm{X}$ & & & \\
\hline Familia Calappidae & & & & & Pentaceraster cumingi & & & & $\mathrm{X}$ \\
\hline Calappa spp. & $\mathrm{X}$ & & & & Clase Echinoidea & & & & \\
\hline Familia Dairidae & & & & & Orden Arbacioida & & & & \\
\hline Daira americana & $\mathrm{X}$ & & & & Familia Arbaciidae & & & & \\
\hline Familia Diogenidae & & & & & Arbacia stellata & & $\mathrm{X}$ & & \\
\hline Aniculus elegans & & $\mathrm{X}$ & & & Orden Camarodonta & & & & \\
\hline Familia Inachoididae & & & & & Familia Toxopneustidae & & & & \\
\hline Stenorhynchus debilis & $\mathrm{X}$ & $\mathrm{X}$ & $\mathrm{X}$ & & Toxopneustes roseus & $\mathrm{X}$ & $\mathrm{X}$ & & \\
\hline Familia Leucosiidae & & & & & Tripneustes depressus & $\mathrm{X}$ & $\mathrm{X}$ & $\mathrm{X}$ & \\
\hline Leucosilia jurinii & & & & $\mathrm{X}$ & Orden Cidaroida & & & & \\
\hline Familia Mithracidae & & & & & Familia Cidaridae & & & & \\
\hline Teleophrys cristulipes & $\mathrm{X}$ & $\mathrm{X}$ & $\mathrm{X}$ & & Eucidaris thouarsii & $\mathrm{X}$ & $\mathrm{X}$ & $\mathrm{X}$ & \\
\hline Familia Palaemonidae & & & & & Orden Diadematoida & & & & \\
\hline Gnathophyllum panamense & & $\mathrm{X}$ & & & Familia Diadematidae & & & & \\
\hline Palaemonella holmesi & & & & $\mathrm{X}$ & Astropyga pulvinata & $\mathrm{X}$ & $\mathrm{X}$ & $\mathrm{X}$ & $\mathrm{X}$ \\
\hline Pontonia margarita & $\mathrm{X}$ & $\mathrm{X}$ & & & Diadema mexicanum & $\mathrm{X}$ & $\mathrm{X}$ & $\mathrm{X}$ & \\
\hline Zenopontonia soror & $\mathrm{X}$ & $\mathrm{X}$ & & $\mathrm{X}$ & Orden Spatangoida & & & & \\
\hline Familia Palinuridae & & & & & Familia Loveniidae & & & & \\
\hline Panulirus gracilis & $\mathrm{X}$ & $\mathrm{X}$ & & & Loveniidae spp. & & & & $X$ \\
\hline Familia Parthenopidae & & & & & Clase Holothuroidea & & & & \\
\hline Parthenopidae sp. & & & & $\mathrm{X}$ & Orden Apodida & & & & \\
\hline Familia Penaeidae & & & & & Familia Synaptidae & & & & \\
\hline Metapenaeopsis kishinouyei & & & & $\mathrm{X}$ & Euapta godeffroyi & $\mathrm{X}$ & & & \\
\hline Familia Pinnotheridae & & & & & Orden Dendrochirotida & & & & \\
\hline Pinnotheridae spp. & & & & $\mathrm{X}$ & Familia Cucumariidae & & & & \\
\hline Familia Porcellanidae & & & & & Cucumaria flamma & $\mathrm{X}$ & $\mathrm{X}$ & $\mathrm{X}$ & \\
\hline Petrolisthes spp. & $\mathrm{X}$ & $\mathrm{X}$ & $\mathrm{X}$ & & Familia Phyllophoridae & & & & \\
\hline Familia Portunidae & & & & & Pentamera chierchiae & $\mathrm{X}$ & & & \\
\hline Portunidae sp. 1 & & & & $\mathrm{X}$ & Orden Holothuriida & & & & \\
\hline
\end{tabular}




\begin{tabular}{llll}
\hline Taxa & AR & AC & BS \\
\hline Familia Holothuriidae & & & \\
Holothuria (Stauropora) fuscocinerea & $\mathrm{X}$ & & \\
Holothuria (Stauropora) pluricuriosa & $\mathrm{X}$ & & \\
Holothuria (Lessonothuria) pardalis & $\mathrm{X}$ & & \\
Holothuria (Mertensiothuria) hilla & $\mathrm{X}$ & & \\
Orden Synallactida & & & \\
Familia Stichopodidae & & & \\
Isostichopus fuscus & $\mathrm{X}$ & $\mathrm{X}$ & \\
Stichopus horrens & $\mathrm{X}$ & & \\
Clase Ophiuroidea & & & \\
Orden Amphilepidida & & & \\
Familia Ophiactidae & & & \\
Ophiactis spp. & $\mathrm{X}$ & $\mathrm{X}$ & $\mathrm{X}$ \\
Familia Ophiotrichidae & & & \\
Ophiothela spp. & $\mathrm{X}$ & $\mathrm{X}$ & $\mathrm{X}$ \\
Ophiothrix spp. & $\mathrm{X}$ & $\mathrm{X}$ & \\
Orden Ophiacanthida & & & \\
Familia Ophiocomidae & & & \\
Ophiocoma alexandri & $\mathrm{X}$ & & \\
Familia Ophiodermatidae & & & \\
Ophioderma spp. & $\mathrm{X}$ & &
\end{tabular}

\section{Filo Chordata}

Clase Ascidiacea

Orden Aplousobranchia

Familia Diazonidae

Rhopalaea birkelandi

Clase Elasmobranchii

Orden Myliobatiformes

Familia Dasyatidae

Hypanus longus

Familia Myliobatidae

Aetobatus laticeps

Rhinoptera steindachneri

Familia Urotrygonidae

Urobatis halleri

Urotrygon chilensis

Urotrygon rogersi

Orden Orectolobiformes

Familia Ginglymostomatidae

Ginglymostoma unami

$\mathrm{X}$

Orden Rajiformes

Familia Rhinobatidae

Pseudobatos prahli

Orden Torpediniformes

Familia Narcinidae

Diplobatis ommata

Clase Actinopterygii

Orden Anguilliformes
Taxa

Familia Muraenidae

Echidna nebulosa

Gymnomuraena zebra

Gymnothorax panamensis

Gymnothorax castaneus

Gymnothorax dovii

Muraena clepsydra

Muraena lentiginosa

Familia Ophichthidae

Myrichthys tigrinus

Orden Aulopiformes

Familia Synodontidae

Synodus lacertinus

X

$\mathrm{X}$

Orden Beryciformes

Familia Holocentridae

Myripristis berndti

Sargocentron suborbitale

$\mathrm{X}$

Orden Perciformes

Familia Acanthuridae

Acanthurus triostegus

Acanthurus xanthopterus

Prionurus laticlavius

AR AC BS FA

Familia Apogonidae

Apogon atricaudus

Apogon dovii

Familia Blenniidae

Ophioblennius steindachneri

Plagiotremus azaleus

X

X X

$\mathrm{X}$

X X

$\mathrm{X}$

$\mathrm{X}$

$\mathrm{X}$

X X

X X X

X

X X

X X

X

X

X X

X $\quad X$

Familia Carangidae

X Alectis ciliaris

X

Caranx caballus

X X

$\mathrm{X} \quad$ Caranx caninus

X Caranx sexfasciatus

Trachinotus rhodopus

Familia Chaetodontidae

X Chaetodon humeralis

Johnrandallia nigrirostris

Cirrhitichthys oxycephalus
Elagatis bipinnulata

Gnathanodon speciosus

Selene spp.

Seriola rivoliana

Familia Chaenopsidae

Acanthemblemaria exilispinus

Acanthemblemaria hancocki

Familia Cirrhitidae

Cirrhitus rivulatus

X X X

$\begin{array}{cc} & \mathrm{X} \\ \mathrm{X} & \mathrm{X} \\ & \mathrm{X} \\ & \mathrm{X} \\ & \mathrm{X} \\ \mathrm{X} & \mathrm{X}\end{array}$

X

$\mathrm{X}$

$\begin{array}{lll}X & X & X\end{array}$

$\mathrm{X} \quad \mathrm{X} \quad \mathrm{X}$

X X

Familia Ephippidae 


\begin{tabular}{|c|c|c|c|c|c|c|c|c|c|}
\hline Taxa & $\mathrm{AR}$ & $\mathrm{AC}$ & BS & FA & Taxa & $\mathrm{AR}$ & $\mathrm{AC}$ & BS & FA \\
\hline Chaetodipterus zonatus & $\mathrm{X}$ & & & $\mathrm{X}$ & Nicholsina denticulata & & & $X$ & \\
\hline Familia Gobiidae & & & & & Scarus ghobban & $\mathrm{X}$ & $\mathrm{X}$ & $\mathrm{X}$ & \\
\hline Coryphopterus urospilus & $\mathrm{X}$ & & & & Scarus rubroviolaceus & $\mathrm{X}$ & & & \\
\hline Elacatinus puncticulatus & $\mathrm{X}$ & $\mathrm{X}$ & & & Familia Serranidae & & & & \\
\hline Tigrigobius digueti & $\mathrm{X}$ & $\mathrm{X}$ & & & Alphestes immaculatus & $\mathrm{X}$ & $\mathrm{X}$ & & \\
\hline Familia Haemulidae & & & & & Cephalopholis panamensis & $\mathrm{X}$ & $\mathrm{X}$ & & \\
\hline Anisotremus interruptus & $\mathrm{X}$ & & & & Epinephelus labriformis & $\mathrm{X}$ & $\mathrm{X}$ & $\mathrm{X}$ & \\
\hline Anisotremus taeniatus & $\mathrm{X}$ & & & & Paranthias colonus & $\mathrm{X}$ & $\mathrm{X}$ & & \\
\hline Haemulon flaviguttatum & $\mathrm{X}$ & $\mathrm{X}$ & $\mathrm{X}$ & & Rypticus bicolor & $\mathrm{X}$ & $\mathrm{X}$ & & $\mathrm{X}$ \\
\hline Haemulon maculicauda & $\mathrm{X}$ & $\mathrm{X}$ & & & Rypticus nigripinnis & & & & $\mathrm{X}$ \\
\hline Haemulon sexfasciatum & $\mathrm{X}$ & & $\mathrm{X}$ & & Serranus psittacinus & & $\mathrm{X}$ & & \\
\hline Familia Kyphosidae & & & & & Familia Sparidae & & & & \\
\hline Kyphosus elegans & & & $\mathrm{X}$ & & Calamus brachysomus & $\mathrm{X}$ & & & \\
\hline Familia Labridae & & & & & Familia Tripterygiidae & & & & \\
\hline Bodianus diplotaenia & $\mathrm{X}$ & $\mathrm{X}$ & $\mathrm{X}$ & & Lepidonectes clarkhubbsi & $\mathrm{X}$ & $\mathrm{X}$ & & \\
\hline Halichoeres chierchiae & $\mathrm{X}$ & $X$ & $\mathrm{X}$ & & Orden Scorpaeniformes & & & & \\
\hline Halichoeres dispilus & $\mathrm{X}$ & $X$ & $\mathrm{X}$ & & Familia Scorpaenidae & & & & \\
\hline Halichoeres nicholsi & $\mathrm{X}$ & $X$ & $\mathrm{X}$ & & Scorpaena mystes & $\mathrm{X}$ & $\mathrm{X}$ & & \\
\hline Halichoeres notospilus & $\mathrm{X}$ & $\mathrm{X}$ & & & Orden Syngnathiformes & & & & \\
\hline Novaculichthys taeniourus & $\mathrm{X}$ & $\mathrm{X}$ & & & Familia Fistulariidae & & & & \\
\hline Thalassoma lucasanum & $\mathrm{X}$ & $\mathrm{X}$ & $\mathrm{X}$ & & Fistularia commersonii & $\mathrm{X}$ & $\mathrm{X}$ & & \\
\hline Familia Labrisomidae & & & & & Familia Syngnathidae & & & & \\
\hline Malacoctenus zonifer & $\mathrm{X}$ & & & & Doryrhamphus excisus & $\mathrm{X}$ & & & \\
\hline Familia Lutjanidae & & & & & Orden Tetraodontiformes & & & & \\
\hline Lutjanus argentiventris & $\mathrm{X}$ & $\mathrm{X}$ & $\mathrm{X}$ & & Familia Balistidae & & & & \\
\hline Lutjanus guttatus & $\mathrm{X}$ & $\mathrm{X}$ & & & Balistes polylepis & $\mathrm{X}$ & $\mathrm{X}$ & & \\
\hline Lutjanus inermis & $\mathrm{X}$ & & & & Pseudobalistes naufragium & $\mathrm{X}$ & $\mathrm{X}$ & $\mathrm{X}$ & \\
\hline Familia Malacanthidae & & & & & Sufflamen verres & $\mathrm{X}$ & & $\mathrm{X}$ & $\mathrm{X}$ \\
\hline Malacanthus brevirostris & & & & $\mathrm{X}$ & Familia Diodontidae & & & & \\
\hline Familia Mullidae & & & & & Chilomycterus reticulatus & & $\mathrm{X}$ & & \\
\hline Mulloidichthys dentatus & $\mathrm{X}$ & & $\mathrm{X}$ & & Diodon holocanthus & $\mathrm{X}$ & $\mathrm{X}$ & $\mathrm{X}$ & \\
\hline Familia Pomacanthidae & & & & & Familia Monacanthidae & & & & \\
\hline Holacanthus passer & $\mathrm{X}$ & $\mathrm{X}$ & & & Aluterus scriptus & & $\mathrm{X}$ & & \\
\hline Pomacanthus zonipectus & $\mathrm{X}$ & $\mathrm{X}$ & & & Familia Ostraciidae & & & & \\
\hline Familia Pomacentridae & & & & & Ostracion meleagris & $\mathrm{X}$ & & & \\
\hline Abudefduf concolor & $\mathrm{X}$ & & & & Familia Tetraodontidae & & & & \\
\hline Abudefduf troschelii & $\mathrm{X}$ & $\mathrm{X}$ & $\mathrm{X}$ & & Arothron meleagris & $\mathrm{X}$ & & $X$ & \\
\hline Chromis atrilobata & $\mathrm{X}$ & $\mathrm{X}$ & & & Arothron hispidus & $\mathrm{X}$ & $\mathrm{X}$ & & \\
\hline Microspathodon dorsalis & $\mathrm{X}$ & $X$ & $\mathrm{X}$ & & Canthigaster punctatissima & $\mathrm{X}$ & $\mathrm{X}$ & $X$ & \\
\hline Stegastes acapulcoensis & $\mathrm{X}$ & & $\mathrm{X}$ & & Sphoeroides lobatus & $\mathrm{X}$ & $\mathrm{X}$ & & \\
\hline Stegastes flavilatus & $\mathrm{X}$ & $\mathrm{X}$ & $\mathrm{X}$ & & TOTALES & 175 & 130 & 64 & 72 \\
\hline
\end{tabular}




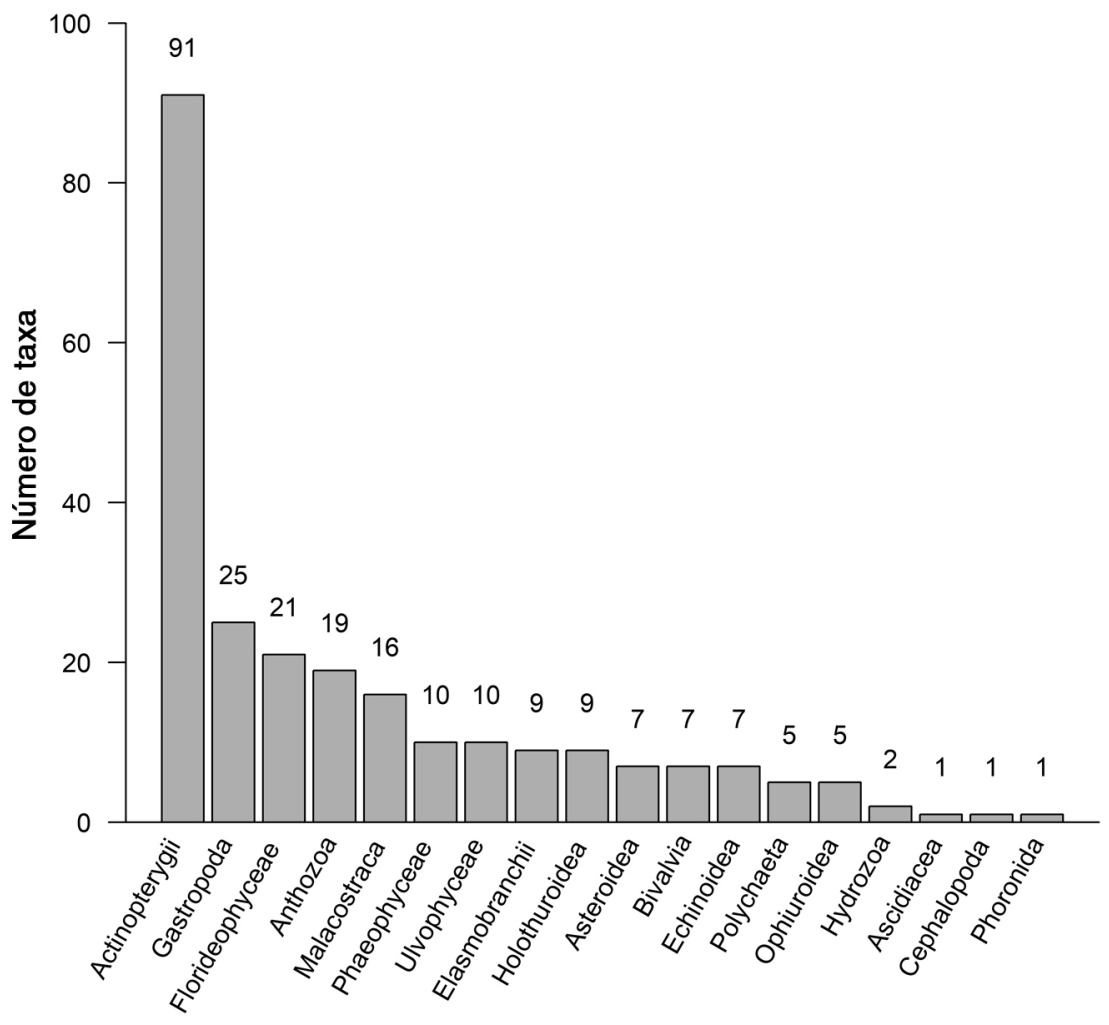

Grupo taxonómico

Fig. 2. Número de taxones observados por grupo taxonómico durante la expedición Santa Elena Waitt-MarViva (2018), Pacífico Norte de Costa Rica.

Fig. 2. Number of taxa observed by taxonomic group during the Waitt-MarViva Santa Elena expedition (2018), North Pacific of Costa Rica.

blandos). Los bosques de Sargazo tuvieron una riqueza de 64 especies, donde los peces dominaron con 28 especies, mientras que los otros grupos presentaron menos de 10 especies. Finalmente, los fondos arenosos tuvieron una riqueza de 72 especies, con una dominancia de peces óseos y batoideos con 21 especies, y al igual que el bosque de Sargazo, los demás grupos fueron representados por menos de 10 especies. A continuación, se presenta una descripción de cada ambiente estudiado.

Arrecifes coralinos (Fig. 4): Se consideraron arrecifes de coral los ambientes cuyo fondo estaba compuesto por basamento coralino principalmente, y los corales crecen sobre el mismo. Los sitios donde se observó dicha característica fueron: Isla Golondrina Sur, Isla Negritos, Isla Pelada Sur, Isla San José Norte, Matapalito y Punta Blanca (Fig. 1). El ámbito de profundidad fue de 3.3-10.5 m. Muchos arrecifes están construidos principalmente por el coral Pavona gigantea. Otras especies de Pavona (P. clavus, $P$. varians, $P$. chiriquiensis) y Psammocora stellata, únicamente fueron observados en arrecifes y comunidades coralinas a lo largo de esta investigación.

El fondo estuvo dominado por tapetes algales en la mayoría de los sitios, con excepción de Isla San José Norte, donde los crustáceos de la infraclase Cirripedia fueron predominantes. Los tapetes de algas están dominados por especies filamentosas de los órdenes de algas rodófitas Ceramiales y Gelidiales. Dentro del grupo 


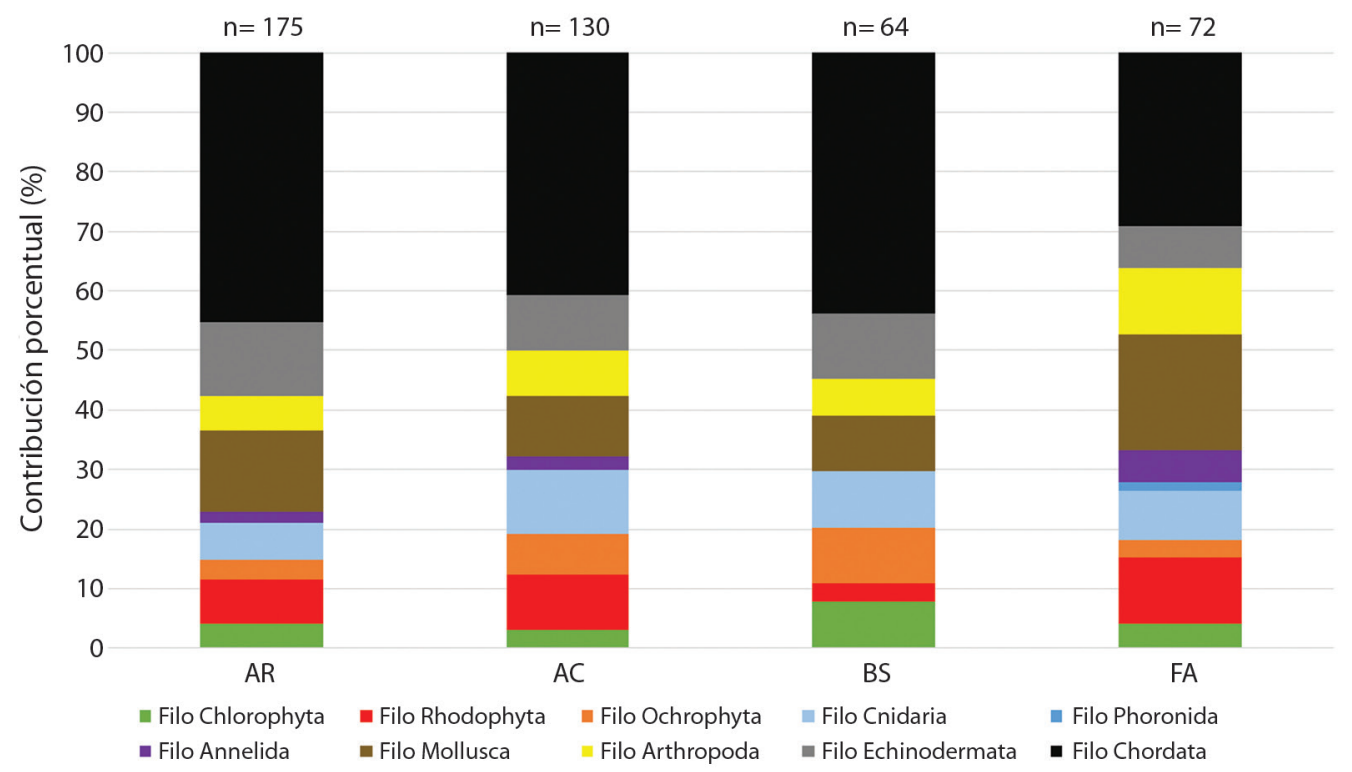

Fig. 3. Contribución porcentual de cada grupo taxonómico a nivel de filo al total de las especies encontradas en cada hábitat submareal estudiado durante la expedición Santa Elena Waitt-MarViva (2018), Pacífico Norte de Costa Rica. AR: Arrecife rocoso; AC: Arrecife de Coral; BS: Bosque de Sargassum; FA: Fondo arenoso. n = \# total de especies por hábitat.

Fig. 3. Percent contribution of each taxonomic group at the phylum level to the total of the species found in each subtidal habitat studied during the Waitt-Marviva Santa Elena expedition (2018), North Pacific of Costa Rica. AR: Rocky reef; AC: Coral Reef; BS: Sargassum Forest; FA: Sandy bottom. n = Total \# of species by habitat.

de las algas se informa por primera vez en esta zona el género Gayliella, también presente en los arrecifes rocosos. Otros organismos también formaron parte de la cobertura del fondo, como anémonas y corales blandos. Algunos invertebrados móviles solo fueron vistos en los arrecifes coralinos (Alpheus lottini, Aniculus elegans, Gnathophyllum panamense, el erizo Arbacia stellata y el bivalvo Pinna rugosa).

Los peces óseos fue el grupo predominante con 52 taxa observados. Al igual que otros grupos, algunas especies sólo fueron observadas en arrecifes coralinos: el muraénido Muraena lentiginosa, los chaenópsidos Acanthemblemaria exilispinus y A. hancocki, el serránido Serranus psittacinus, el diodóntido Chilomycterus reticulatus y el monacántido Aluterus scriptus.

Arrecifes rocosos (Fig. 5): Los hábitats más comúnmente observados fueron los arrecifes rocosos, principalmente alrededor de las puntas, islotes y en bajos conocidos de la zona como Bajo La Vita y Bajo Negro. Los arrecifes rocosos corresponden al ambiente con mayor riqueza de especies. En estos ambientes la cobertura estuvo dominada por los tapetes de algas, seguidos por las algas calcáreas costrosas del orden Corallinales y algas costrosas del orden Peyssonneliales. Otros componentes comunes, pero no dominantes fueron los corales, las macroalgas, las esponjas, los cirripedios y las ascidias. En algunos de estos arrecifes se pudieron observar algunas colonias aisladas de coral (principalmente de Pavona gigantea, Porites lobata y Pocillopora spp.), formaciones de Tubastrea coccinea, y también varias colonias de octocorales como Leptogorgia alba y Carijoa riisei, y en algunos casos formaciones relativamente extensas de coral negro (Anthipates spp.), como en Bajo Negro. 

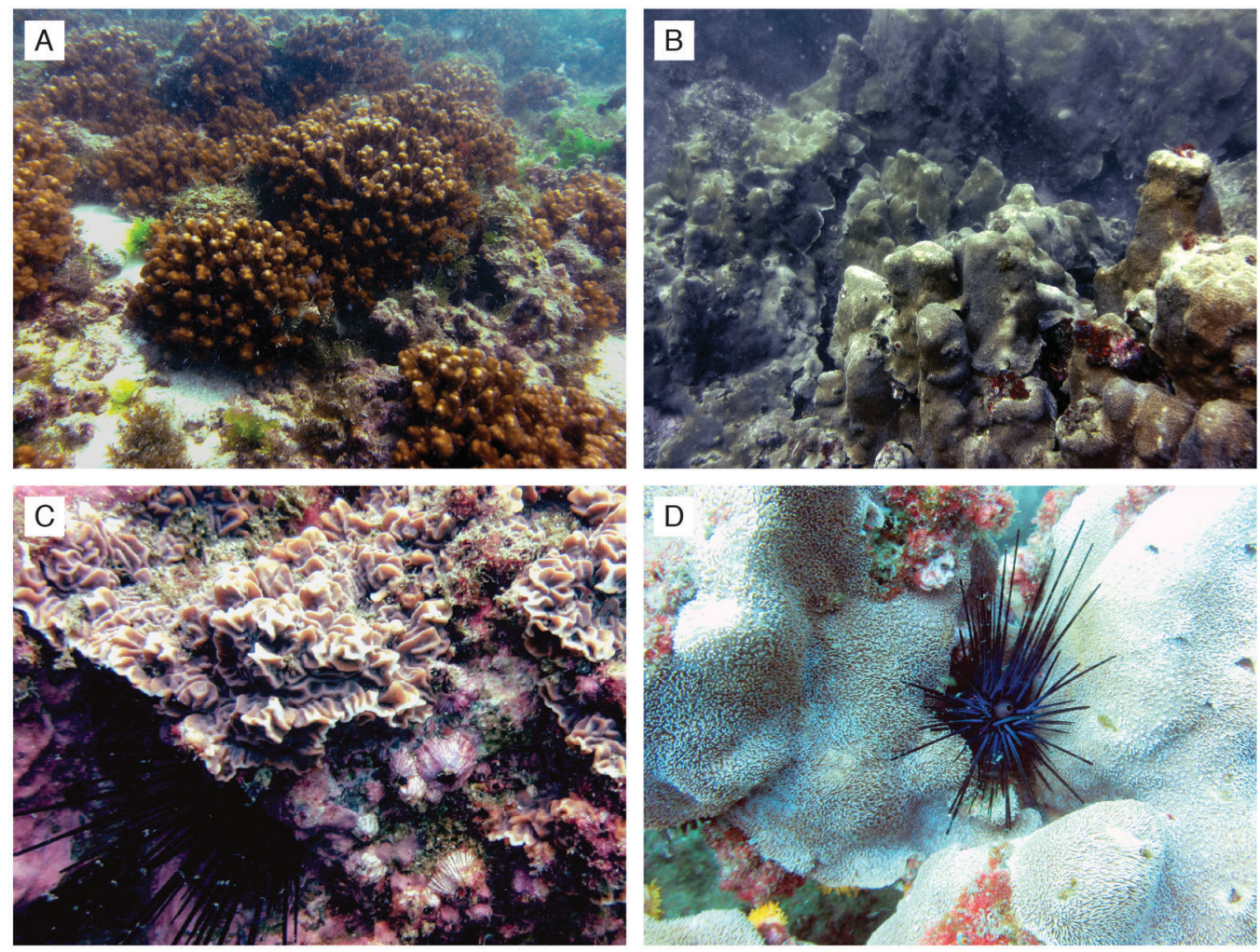

Fig. 4. Arrecifes coralinos. A) Arrecife dominado por Pocillopora spp. en Matapalito; B). coral formador de arrecife Pavona clavus; C) basamento coralino cubierto por Pavona varians; D) el erizo de mar Diadema mexicanum entre colonias de Pavona gigantea.

Fig. 4. Coral reefs. A) Coral reef dominated by Pocillopora spp. in Matapalito; B) coral reef formed by the coral Pavona clavus; C) coral framework covered by Pavona varians; D) sea urchin Diadema mexicanum among colonies of Pavona gigantea.

Asimismo, es común observar talos de algas como Dictyota, Padina, Amphiroa, Asparagopsis (tetraesporófito), Hypnea y Gracilaria.

En los arrecifes rocosos también fue posible encontrar una gran diversidad de invertebrados móviles, particularmente erizos de mar (con predominancia de Diadema mexicanum). En cuanto a los ensambles de peces, estos fueron bastante diversos y heterogéneos, donde era posible apreciar la dominancia de cardúmenes de piscívoros, principalmente pargos (Lutjanus argentiventris y Lutjanus guttatus) y roncadores (Haemulon flaviguttatum, Haemulon maculicauda y Haemulon sexfasciatum).

En dos de los arrecifes rocosos, Puertas de La Iglesia y Bajo La Vita, observamos colonias de poliquetos tubícolas sobre los promontorios rocosos, con un contorno esférico de 20-40 $\mathrm{cm}$ de diámetro, conformadas por estructuras rígidas ramificadas. La especie formadora de estas colonias es Salmacina tribranchiata, de la familia Serpulidae. En el campo fue posible observar que las estructuras construidas por las colonias de $S$. tribranchiata proveían también superficies para el crecimiento de los tapetes de algas, y refugio para algunos invertebrados (como cangrejos ermitaños y gasterópodos), e incluso algunos peces pequeños, los cuales se escondían entre las ramificaciones de las colonias cuando los buzos se aproximaban.

Bosques de Sargassum (Fig. 6): Se encontraron dos sitios alrededor de Isla Colorada (Fig. 1) con alta cobertura de Sargassum. 

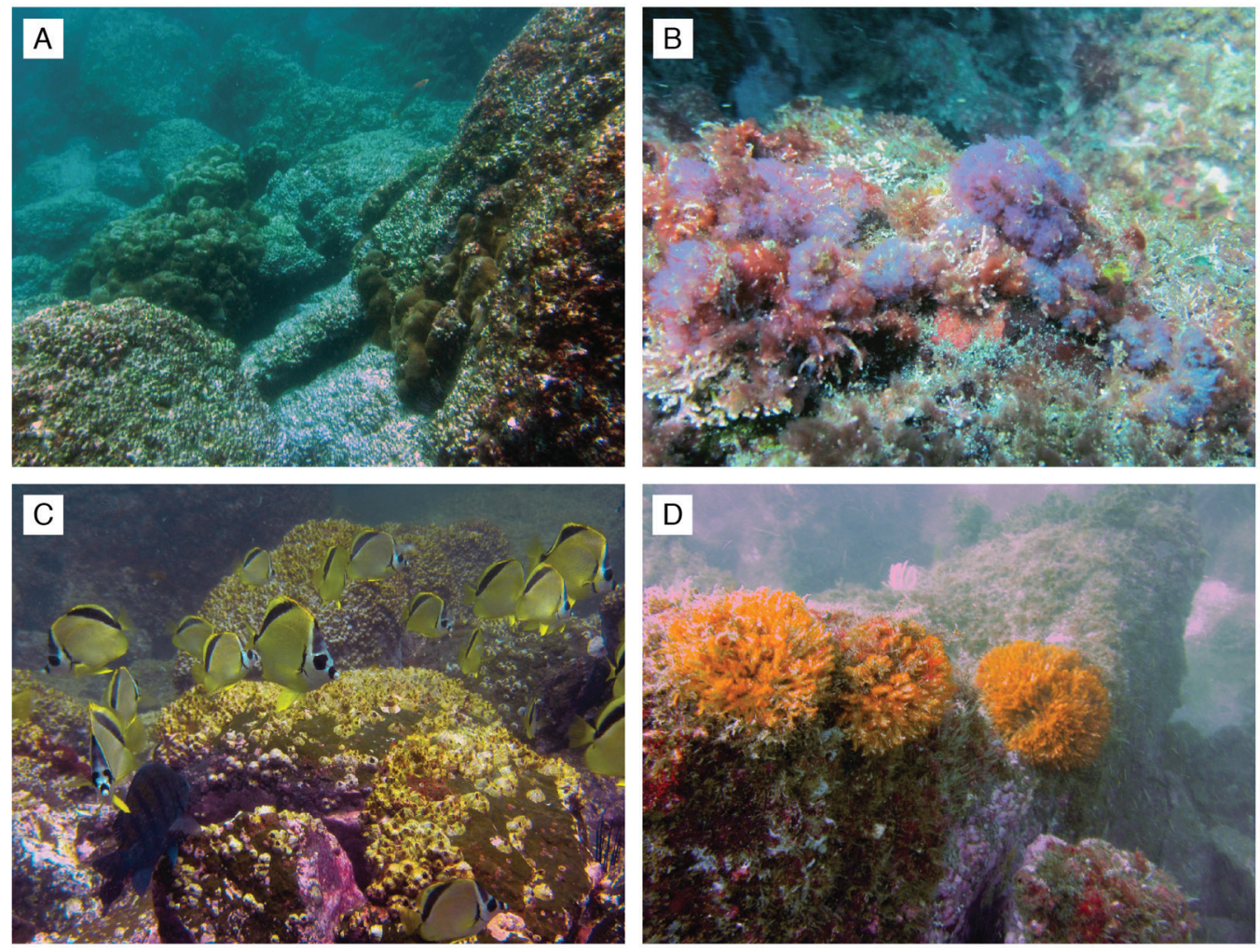

Fig. 5. Arrecifes rocosos. A) Colonias coralinas aisladas en medio de las rocas; B) tapetes de algas rojas y verdes cubriendo el sustrato rocoso; C) el pez Johnrandallia nigrirostris en un arrecife rocoso cubierto con cirripedios y algas rojas calcáreas; D) colonias del poliqueto Salmacina tribranchiata.

Fig. 5. Rocky reefs. A) Isolated coral colonies in the middle of the rocks; B) red and green algae mats covering the rocky substrate; C) the fish Johnrandallia nigrirostris on a rocky reef covered with barnacles and calcareous red algae; D) colonies of the polychaete Salmacina tribranchiata.

Cortés et al. (2014), mencionan que estos bosques están compuestos por la especie Sargassum liebmanni. Sin embargo, al revisar el material de este trabajo se encontraron dos morfologías que podrían referir a dos especies diferentes que están entremezcladas. Para este trabajo se manejó solo una especie ya que se requieren análisis moleculares y morfológicos en más detalle para esclarecer la taxonomía de este género en la región. En comparación con otros sitios de estudio, en los bosques de Sargassum, la presencia de pulpos y gasterópodos fue mayor. Asimismo, se encontraron otras especies de macroalgas asociadas y espacios de arena como elementos predominantes (Fig. 6).

Planicies arenosas (Fig. 7): Diferentes sitios presentaron planicies arenosas, a saber, Matapalito profundo, Isla Las Golondrinas, Punta Santa Elena, Punta Pochote y Punta Blanca (Fig. 1). Los fondos arenosos muestreados se encontraron en un ámbito de profundidad entre los 5 y los $35 \mathrm{~m}$ de profundidad. Para este tipo de hábitat, se utilizaron diferentes metodologías, entre ellas el uso de dragas, las cuales aportan una idea de la macrofauna 

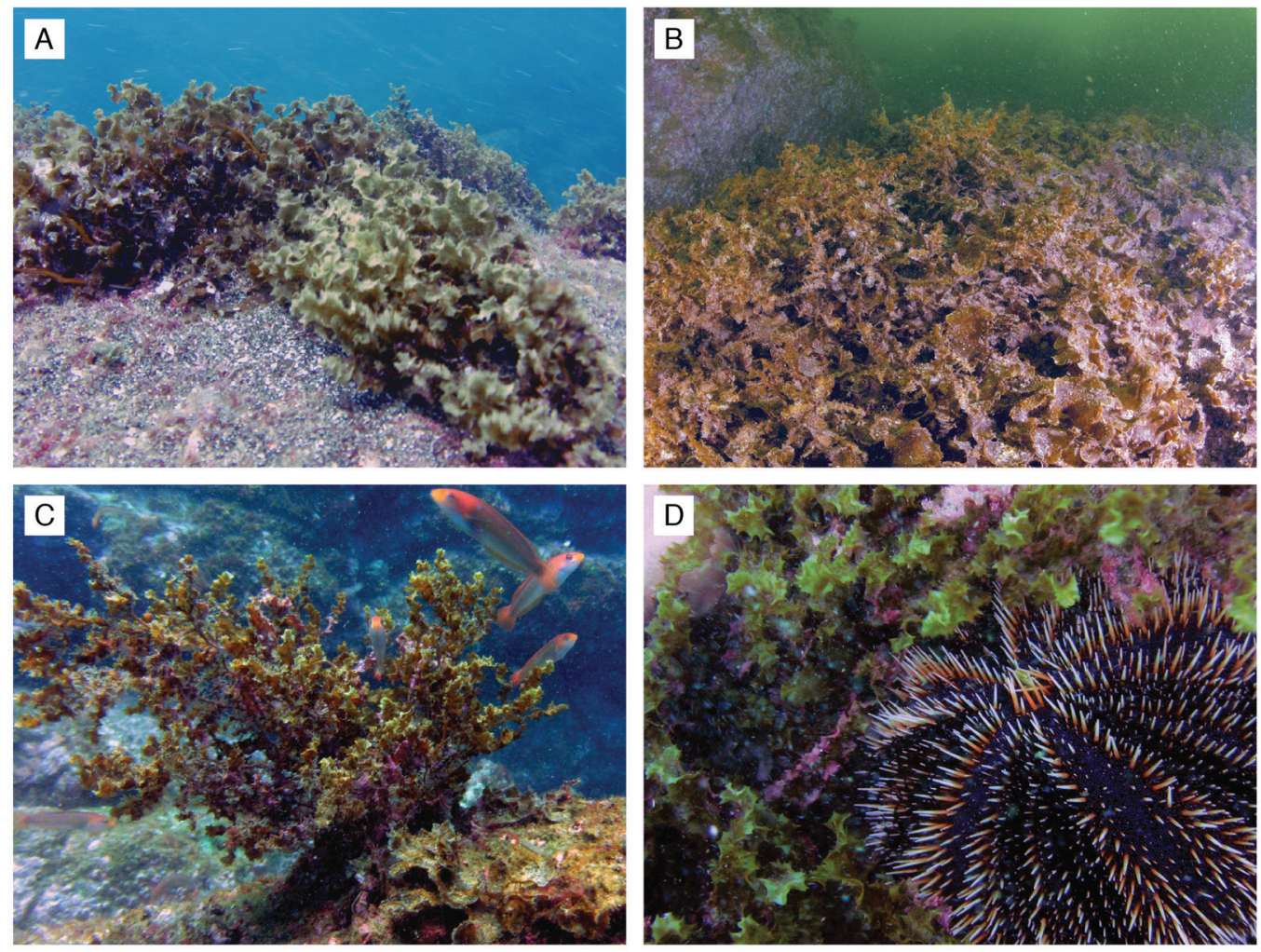

Fig. 6. Bosques de Sargassum. A) Espacios de arena entre los bosques de Sargassum; B) Sargassum liebmannii mezclado con otras macroalgas como Padina y Dictyota; C) hábitat de S. liebmanii en zona rocosa; D) Tripneustes depressus ramoneando entre las macroalgas.

Fig. 6. Sargassum forests. A) sand spaces between Sargassum forests; B) Sargassum liebmannii mixed with other macroalgae such as Padina and Dictyota; C) habitat of S. liebmanii in rocky area; D) Tripneustes depressus browsing among macroalgae.

presente en cada sitio. Otros de los métodos utilizados, fueron censos visuales y recolectas errantes, lo que nos permite conocer puntualmente la riqueza de especies.

El filo Chordata fue el más representado para este ambiente, debido al tipo de muestreo. Las especies de cordados que más se observaron en los fondos arenosos fueron Urobatis halleri, Diplobatis ommata y Ripticus bicolor. Entre los invertebrados más comunes se observaron Pentaceraster cumingi, Zenopontonia soror (asociado a las diferentes especies de estrellas de mar) y Astropyga pulvinata (Fig. 7). Uno de los invertebrados sésiles más conspicuos que se reportan para este ambiente son las anémonas tubícolas de la familia Cerianthidae, que corresponde al primer registro para el país.
Sin embargo, por dificultades en la capacidad de identificación no se reporta por ahora la especie (Fig. 7).

Por otro lado, el filo con menos representación en la investigación fue Phoronida, con una especie, Phoronopsis albomaculata. Estos invertebrados son un grupo pequeño, integrado solo por 11 especies y dos géneros (Santagata, 2015). Phoronopsis albomaculata, se reporta por primera vez para la costa costarricense. Anteriormente había sido informada únicamente en la Isla del Coco (Dean, Sibaja-Cordero, \& Cortés, 2009).

Con respecto a las macroalgas, se encontró que hay especies muy particulares que no se encuentran en ninguno de los otros ambientes aquí estudiados. Este es el caso del nuevo 

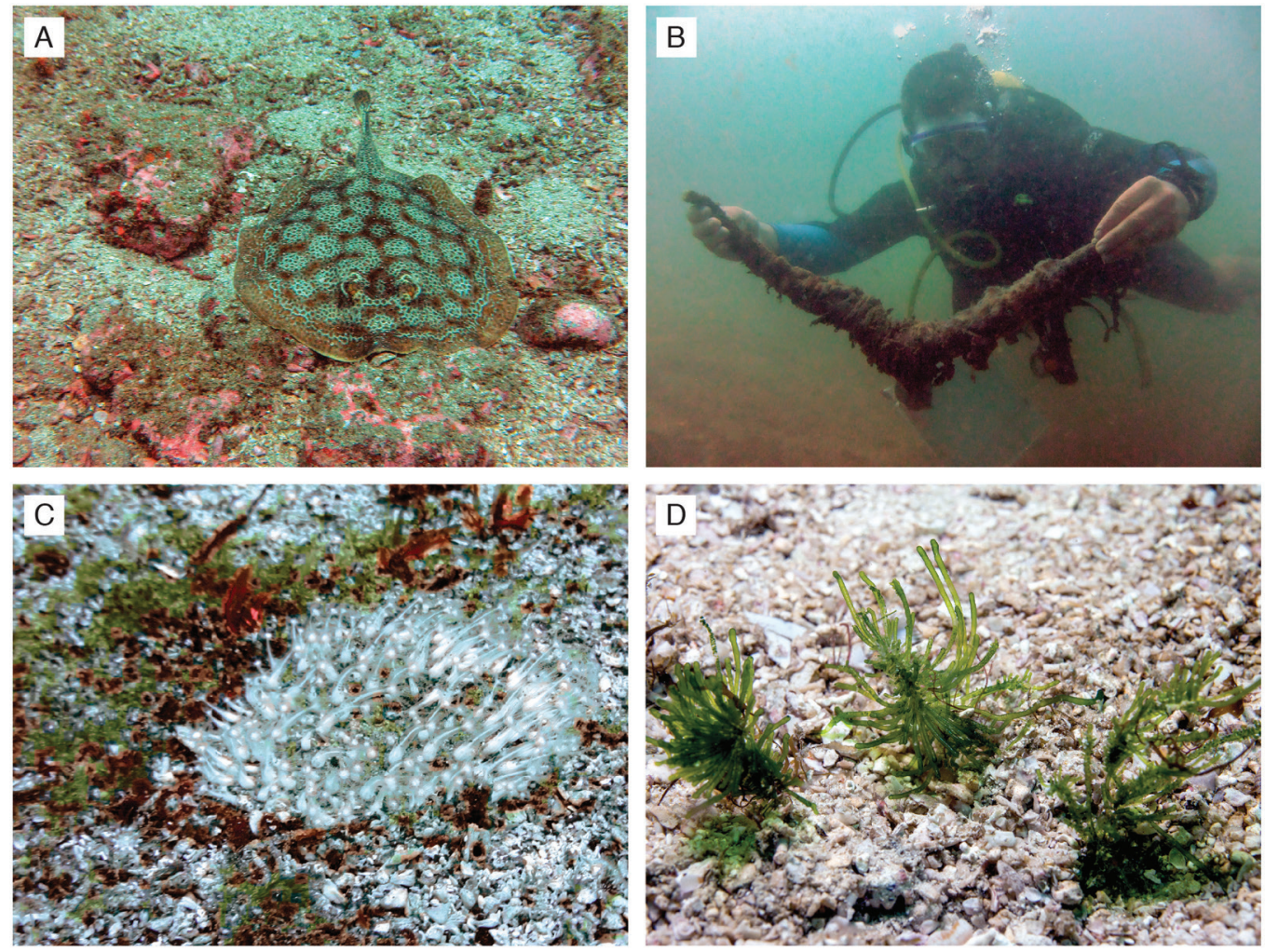

Fig. 7. Planicies arenosas. A) Raya redonda Urobatis halleri sobre sustrato arenoso; B) buzo recolectando la anémona tubícola de la familia Cerianthidae; C) pensamiento de mar, Renilla sp.; D) nuevo reporte de alga verde del género Siphonocladus sp.

Fig. 7. Sandy plains. A) Round ray Urobatis halleri on sandy substrate; B) diver collecting the tube anemone of the family Cerianthidae; C) the Sea pansy, Renilla sp.; D) new report of green algae of the genus Siphonocladus sp.

reporte del alga verde Siphonocladus spp. para Costa Rica. Este género sólo había sido reportado en el Pacífico de América en dos islas de México (Isla Socorro parte de las Islas Revillagigedo y en la Isla Guadalupe) (Pedroche, Aguilar-Rosas, Dreckmann, \& Aguilar-Rosas, 2005). Por lo que este representa una ampliación de rango del género en aguas tropicales.

Por otro lado, en estos ambientes arenosos de profundidad destacan las algas rojas de los géneros Hypoglossum, Halymenia, Ceramium, Scinaia spp. y Chondria platyclada y las algas pardas del género Dictyota. Todas estas algas se observan en este ambiente en alturas superiores (más de $7 \mathrm{~cm}$ ) de lo que se encuentran en los arrecifes de coral y zonas rocosas.

\section{DISCUSIÓN}

Cortés (2017) hace referencia al desconocimiento y a los vacíos de información sobre la biodiversidad marina para el ACG en comparación con otras localidades del Pacífico costarricense. No obstante, en los últimos años el Proyecto BioMar-ACG (Cortés, 2017) ha realizado nuevos aportes a la diversidad marina de esta zona (Cortés \& Joyce, 2020; Vargas \& Cortés, 2019). Todos los hábitats que se trataron en esta investigación ya habían sido 
citados en trabajos previos (Cortés \& Joyce, 2020; Vargas \& Cortés, 2019); sin embargo, pocos trabajos describen la historia natural y diversidad específica para los mismos.

Muchos arrecifes coralinos del ACG están construidos principalmente por el coral Pavona gigantea. Esta especie de coral masivo provee estructuras tridimensionales densas que sirven de hábitat para numerosas especies, lo que ha contribuido con el mantenimiento a largo plazo de las comunidades arrecifales (Tortolero-Langarica, Carricart-Ganivet, Cupul-Magaña, \& Rodríguez-Troncoso, 2017). Algunas especies de peces únicamente fueron observadas en arrecifes coralinos, esto evidencia la importancia que tienen estos ambientes como reservorio de ciertas especies (Riginos \& Leis, 2019). Los arrecifes coralinos son hábitats muy complejos y cambiantes; en las últimas décadas se ha resaltado la importancia de conservar las funciones críticas de estos ecosistemas, como la formación de hábitat y otras formas de mantenimiento de la biodiversidad (Bellwood, Streit, Brandl, \& Tebbett, 2019).

En estudios realizados por Alvarado et al. $(2011,2015,2016,2018,2020)$ en arrecifes del Pacífico, se ha observado que, por lo general, las algas tienden a tener un tamaño menor a $10 \mathrm{~cm}$ de alto y suelen formar tapetes. Sin embargo, en la zona norte de Costa Rica es común encontrar macroalgas de más de 10 $\mathrm{cm}$ de altura. En particular, se han encontrado bosques submarinos estacionales conformados por el alga parda del género Sargassum; estos bosques fueron descritos por primera vez en la Isla Bolaños, Bahía Salinas, al norte de nuestra zona de estudio (Cortés et al., 2014). En toda la zona de estudio es común encontrar algas de este género que se mezclan con otras algas como Dictyota y Padina. Sin embargo, se encontraron dos sitios alrededor de Isla Colorada, donde la cobertura de este género es alta (Alvarado et al., en prensa).

Los bosques de Sargassum presentan estacionalidad a lo largo del año, de manera que son abundantes en la época de afloramiento de aguas frías ricas en nutrientes (Cortés et al., 2014; Stuhldreier et al., 2015). Ecológicamente estos bosques destacan debido a que son zonas de criaderos de juveniles de peces, zonas de alimentación y refugio de herbívoros (Cheminée et al., 2017; Suárez et al., 2021). Debido a su estacionalidad, importancia ecológica, y al hecho de que el reporte de S. liebmanni por Cortés et al. (2014) para los bosques de estas algas en el Pacífico norte se podría tratar de diferentes especies, este ecosistema necesita ser estudiado con más detalle.

Dos de los nuevos reportes que se presentan en este estudio provienen de planicies arenosas, demostrando que se trata de un ambiente aún poco estudiado. Las planicies arenosas son ambientes muy cambiantes en cuanto a la biodiversidad, según las particularidades que presente cada sitio. Algunos de los factores más influyentes en marcar las diferencias en cuanto a composición son: características de corriente, de granulometría y de entrada de luz. A nivel de observación, el grosor y la composición del sustrato varió entre los sitios incluidos en esta investigación, sin embargo, estos datos no formaron parte de nuestro estudio.

Este estudio presenta información sobre las planicies arenosas a partir del uso de diferentes metodologías, una de ellas el uso de dragas para conocer la macrofauna. En sustratos blandos, la macrofauna se ha estudiado desde diferentes enfoques, entre ellos midiendo su aporte en la red trófica, así como bioindicadores de salud del ecosistema (Dauvin et al., 2017). Los fondos arenosos son importantes sitios de alimentación de batoideos y otros peces, tienen una gran influencia en el ciclo de nutrientes y de materia orgánica, y además funcionan como sitios de descanso de especies grandes (Dauvin et al., 2017; Link, 2001; Reiss \& Kroncke, 2005). La fauna bentónica asociada a sustratos blandos funciona como bioindicador, a medida que se relaciona con las características del entorno, como por ejemplo poliquetos de las familias Capitellidae y Spionidae (Dean, 2008). Estos organismos presentan poca movilidad y en algunos casos ciclos de vida largos, teniendo una estrecha relación con el sedimento, lo que los convierte en organismos modelo para la evaluación de perturbaciones y cambios 
ambientales (Clarke \& Warwick, 1994; Frithsen \& Holland, 1990). Otro de los métodos utilizados en las planicies arenosas fueron censos visuales y recolectas errantes, permitiendo conocer puntualmente la riqueza de especies. Sin embargo, sería enriquecedor para futuros estudios investigar anualmente los cambios en la diversidad debido al afloramiento costero (Jiménez, Cortés, León, \& Ruiz, 2001), ya que las características abióticas que presentan las planicies arenosas tienen un efecto directo en la biodiversidad específica asociada a estos ambientes (Gray, 1981).

Conclusiones: El ACG es un sitio que presenta una gran biodiversidad marina. Durante esta expedición, se encontraron cuatro nuevos reportes para el país, entre ellos el reporte de la subclase Ceriantharia, dos nuevos géneros de algas (el alga roja Gaylliela spp. y alga verde Siphonocladus spp.) y la especie Scinaia complanata. Es esperable que con un mayor esfuerzo de muestreo, que abarque un periodo más extenso, se pueda registrar mayor riqueza de especies para esta zona.

La Península de Santa Elena y las Islas Murciélago presentan ecosistemas muy particulares, como las comunidades coralinas donde predomina el género Pavona, arrecifes rocosos cubiertos principalmente con algas calcáreas, colonias de poliquetos, bosques de Sargassum y planicies arenosas con características abióticas muy cambiantes. Estos ecosistemas se encuentran expuestos al afloramiento costero, de manera que los cambios en las corrientes, la temperatura o el ingreso de agua dulce, pueden ocasionar cambios en la composición de especies.

El valor de este estudio, al igual que otros estudios de biodiversidad, se centra en el registro histórico de los organismos. Estos registros pueden ser aprovechados para definir vínculos empíricos entre rasgos funcionales y las funciones ecosistémicas que permitan mejorar las estrategias de conservación (Bellwood et al., 2019). El registro de la biodiversidad y sus cambios a través del tiempo son fundamentales para justificar y diseñar estrategias de conservación que impulsen el desarrollo de forma sostenible.

Declaración de ética: los autores declaran que todos están de acuerdo con esta publicación y que han hecho aportes que justifican su autoría; que no hay conflicto de interés de ningún tipo; y que han cumplido con todos los requisitos y procedimientos éticos y legales pertinentes. Todas las fuentes de financiamiento se detallan plena y claramente en la sección de agradecimientos. El respectivo documento legal firmado se encuentra en los archivos de la revista.

\section{AGRADECIMIENTOS}

La expedición Santa Elena se realizó gracias a la organización de la Fundación MarViva y el financiamiento de Waitt Foundation y Waitt Institute. Agradecemos especialmente el trabajo de Erick Ross y Mónica Espinoza de Fundación MarViva. Asimismo, este trabajo fue posible gracias a la colaboración de Joe Lepore, Paul Robinson, Joey Baxter, Benjamin Barrie Todd y toda la tripulación del Plan B. Le agradecemos especialmente a Diego Mejías por todo el apoyo brindado durante la expedición, por la fotografía y video de los especímenes y ambientes. Extendemos el agradecimiento al equipo de Misión Tiburón y de Tora Carey. El trabajo se realizó con los permisos del Área de Conservación Guanacaste (ACG) R-SINAC-ACG-PI-022-2018.

\section{RESUMEN}

Introducción: Los hábitats costeros de todo el mundo están experimentando una presión cada vez mayor debido a la contaminación, el desarrollo costero, la pesca y el cambio climático. Identificar y registrar la biodiversidad costera es esencial para evaluar la salud, los cambios y el alcance de la pérdida de biodiversidad de los ecosistemas. Los hábitats costeros tropicales como los arrecifes de coral y los lechos de pastos marinos han sido el foco de investigación de los científicos durante las últimas décadas; sin embargo, se han descuidado otros ecosistemas, como los bosques de macroalgas, los fondos de sedimentos submareales (20-30 $\mathrm{m}$ de profundidad) y los arrecifes rocosos. 
Objetivo: Este trabajo reporta la biodiversidad marina asociada con arrecifes rocosos, arrecifes de coral, bosques de Sargazo y fondos submareales sedimentarios (20-30 m de profundidad), en un área tropical de afloramiento estacional (Península de Santa Elena, Bahía de Santa Elena e Islas Murciélago, Costa Rica).

Métodos: Durante la Expedición Santa Elena (21 de abril2 de mayo de 2018), se visitaron un total de 28 sitios para registrar la biodiversidad en cuatro hábitats diferentes (arrecifes rocosos y de coral, bosques de sargazo y fondos de sedimentos submareales), utilizando SCUBA, en muestreos sistemáticos, evaluaciones visuales y en algunos fondos de sedimentos se utilizó una draga de arrastre por 20 minutos.

Resultados: Se identificaron un total de 254 taxones, siendo los peces óseos el grupo más diverso con 91 especies, seguido de los gasterópodos (25 spp.), las algas rojas (21 spp.) y los antozoos (19 spp.). Se reportan cuatro nuevos registros para el Pacífico continental de Costa Rica, incluida la subclase de una anémona tubícola (Ceriantharia).

Conclusiones: Los resultados muestran que la península de Santa Elena, la bahía de Santa Elena y las islas Murciélago albergan hábitats poco comunes y no estudiados, como bosques del alga parda Sargassum, fondos de sedimentos, arrecifes construidos por el coral masivo Pavona gigantea, que es muy inusual, y comunidades de arrecifes rocosos dominadas por colonias calcáreas de poliquetos (Salmacina tribranchiata).

Palabras clave: arrecifes de coral; Pacífico Tropical Oriental; bosque de sargazo; fondos arenosos; Área de Conservación Guanacaste.

\section{REFERENCIAS}

Alvarado, J. J., Azofeifa-Solano, J. C., Beita-Jiménez, A., Cortés, J., Mena, S., Salas-Moya, C., \& Fernández-García, C. (En prensa). Reef environments of Murciélago Islands and Santa Elena Peninsula, Guanacaste Conservation Area, Costa Rican Pacific. Scientia Insularum. https://doi.org/10.25145/j. SI.2021.04.004

Alvarado, J. J., Beita-Jimenez, A., Mena, S., FernándezGarcía, C., Cortés, J., Sánchez-Noguera, C., Jimenez, C., \& Guzmán-Mora, A. G. (2018). Cuando la conservación no puede seguir el ritmo del desarrollo: Estado de salud de los ecosistemas coralinos del Pacífico Norte de Costa Rica. Revista de Biología Tropical, 66(Suplemento 1), S280-S308.

Alvarado, J. J., Beita-Jiménez, A., Mena, S., FernándezGarcía, C., \& Guzmán-Mora, A. G. (2015). Ecosistemas coralinos del Área de Conservación Osa, Costa Rica: estructura y necesidades de conservación. Revista de Biología Tropical, 63(Suplemento 1), S219-S259.
Alvarado, J. J., Beita, A., Mena, S., Fernández-García, C., Guzman-Mora, A. G., \& Cortés, J. (2016). Ecosistemas coralinos del Parque Nacional Isla del Coco, Costa Rica: estructura y comparación 1987-2014. Revista de Biología Tropical, 64(Suplemento 1), S153-S175.

Alvarado, J. J., Cortés, J., Esquivel, M. F., \& Salas, E. (2012). Costa Rica's Marine Protected Areas: status and perspectives. Revista de Biología Tropical, 60(1), 129-142.

Alvarado, J. J., Herrera, B., Corrales, L., Asch, J., \& Paaby, P. (2011). Identificación de las prioridades de conservación de la biodiversidad marina y costera en Costa Rica. Revista de Biología Tropical, 59(2), 829-842.

Alvarado, J. J., Sánchez-Noguera, C., Arias-Godínez, G., Araya, T., Fernández-García, C., \& Guzmán, A. G. (2020). Impact of El Niño 2015-2016 on the coral reefs of the Pacific of Costa Rica: the potential role of marine protection. Revista de Biología Tropical, 68(Supplement 1), S271-S282.

Bellwood, D. R., Streit, R. P., Brandl, S. J., \& Tebbett, S. B. (2019). The meaning of the term 'function' in ecology: a coral reef perspective. Functional Ecology, 33(6), 948-961.

BIOMARCC-SINAC-GIZ. (2012). Clasificación sistemas marino costeros costa pacífica de Costa Rica. San José, Costa Rica.

Breedy, O., \& Guzmán, H. M. (2003). Octocorals from Costa Rica: The genus Pacifigorgia (Coelenterata: Octocorallia: Gorgoniidae). Zootaxa, 281, 1-60.

Chacón-Monge, J. L., Azofeifa-Solano, J.C., Alvarado, J. J., \& Cortés, J. (2021). Área de Conservación Guanacaste Echinoderms, North Pacific of Costa Rica. Revista de Biología Tropical, 69(Supplement 1), S487-S500.

Cheminée, A., Pastor, J., Bianchimani, O., Thiriet, P., Sala, E., Cottalorda, J. M., Jean-Marie Dominici, J. M., Lejeune, P., \& Francour, P. (2017). Juvenile fish assemblages in temperate rocky reefs are shaped by the presence of macro-algae canopy and its threedimensional structure. Scientific Reports, 7(1), 1-11.

Clarke, K. R., \& Warwick, R. M. (1994). Change in Marine Communities: An Approach to Statistical Analysis and Interpretation. Plymouth, UK: Natural Environmental Research Council.

Cortés, J. (2016). The Pacific coastal and marine ecosystems. In M. Kappelle (Ed.), Costa Rican Ecosystems (pp. 97-138). Chicago and London: University of Chicago Press.

Cortés, J. (2017). Marine biodiversity baseline for Área de Conservación Guanacaste, Costa Rica: published records. ZooKeys, (652), 129-127. 
Cortés, J., \& Joyce, F. (2020). BioMar-ACG: A successful partnership to inventory and promulgate marine biodiversity. Biotropica, 52(6), 1103-1106.

Cortés, J., Samper-Villareal, J., \& Bernecker, A. (2014) Seasonal phenology of Sargassum liebmannii J. Agardh (Fucales, Heterokontophyta) in an upwelling area of the Eastern Tropical Pacific. Aquatic Botany, $119,105-110$.

Costello, M. J., Michener, W. K., Gahegan, M., Zhang, Z. Q., \& Bourne, P. E. (2013). Biodiversity data should be published, cited, and peer reviewed. Trends in Ecology \& Evolution, 28(8), 454-461.

Dauvin, J. C., Lucas, S., Navon, M., Lesourd, S., Mear, Y., Poizot, E., \& Alizier, S. (2017). Does the hydrodynamic, morphometric and sedimentary environment explain the structure of soft-bottom benthic assemblages in the Eastern Bay of Seine (English Channel)? Estuarine, Coastal and Shelf Science 189, 156-172.

Dean, H. K. (2008). The use of polychaetes (Annelida) as indicator species of marine pollution: a review. Revista de Biología Tropical, 56(Supplement 4), S11-S38.

Dean, H., Sibaja-Cordero, J., \& Cortés, J. (2009). Occurrence of the Phoronid Phoronopsis albomaculata in Cocos Island, Costa Rica. Pacific Science, 64, 459-462.

Denyer, P., Cortés, J., \& Cárdenes, G. (2005). Hallazgo de dunas fósiles de final del pleistoceno en las Islas Murciélago, Costa Rica. Revista Geológica de América Central, 33, 29-44.

Denyer, P., \& Gazel, E. (2009). The Costa Rican Jurassic to Miocene oceanic complexes: Origin, tectonics and relations. Journal of South American Earth Sciences, $28,429-422$

Frithsen, J. B., \& Holland, A. S. (1990). Benthic communities as indicators of ecosystem condition. In D. H. McKenzie, D. E. Hyatt, \& V. J. McDonald (Eds.), Geological Indicators (pp. 459-460). London, UK: Chapman \& Hall.

Gouvêa, L. P., Assis, J., Gurgel, C. F., Serrão, E. A., Silveira, T. C., Santos, R., Duarte, C. M., Peres, L. M. C., Carvalho, V. F., Batista, M. Bastos, E., Sissini, M. N., \& Horta, P. A. (2020). Golden carbon of Sargassum forests revealed as an opportunity for climate change mitigation. Science of the Total Environment, 729, 138745 .

Gray, J. S. (1981). The Ecology of Marine Sediments. Cambridge, UK: Cambridge University Press.

Hauff, F., Hoernle, K., Van Den Bogaard, P., Alvarado, G., \& Garbe-Shonberg, D. (2000). Age and geochemistry of basaltic complexes in western Costa Rica: Contributions to the geotectonic evolution of Central America. Geochemistry, Geophysics,
Geosystems, 1(1), 1999GC000020. https://doi. org/10.1029/1999GC000020

He, Q., \& Silliman, B. R. (2019). Climate change, human impacts, and coastal ecosystems in the Anthropocene. Current Biology, 29(19), R1021-R1035.

Jiménez, C., Cortés, J., León, A., \& Ruiz, E. (2001). Coral bleaching and mortality associated with the 1997-98 El Niño in an upwelling environment in the eastern Pacific (Gulf of Papagayo, Costa Rica). Bulletin of Marine Science, 69, 151-169.

Legeckis, R. (1988). Upwelling off the Gulfs of Panama and Papagayo in the tropical Pacific during March 1985. Journal of Geophysical Research, 93, 15485-15489.

Link, J. (2001). Ecological considerations in fisheries management. When does it matter? Fisheries, 27(4), 10-16.

Lizano, O. G., \& Alfaro, E. J. (2014). Características de la dinámica atmosférica y oceánica en algunas regiones del Área de Conservación Guanacaste (ACG), Costa Rica. Revista de Biología Tropical, 62(Supplement 4), S17-S31.

Mecho, A., Easton, E. E., Sellanes, J., Gorny, M., \& Mah, C. (2019). Unexplored diversity of the mesophotic echinoderm fauna of the Easter Island ecoregion. Marine Biology, 166(7), 91.

McCreary, J. P., Lee, H. S., \& Enfield, D. B. (1989). The response of the coastal ocean to strong offshore winds, with application to the Gulfs of Tehuantepec and Papagayo. Journal of Marine Research, 47, 81-109.

Pedroche, F. F., Silva, P. C., Aguilar-Rosas, L. E., Dreckmann, K. M., \& Aguilar-Rosas, R. (2005). Catálogo de las algas marinas bentónicas del Pacífico de México. I. Chlorophycota. Ensenada, México: Universidad Autónoma de Baja California.

Reiss, H., \& Kroncke, I. (2005). Seasonal variability of benthic indices: an approach to test the applicability of different indices for ecosystem quality assessment. Marine Pollution Bulletin, 50(12), 1490-1499.

Riginos, C., \& Leis, J. M. (2019). Do tiny fish rule the reefs? Science, 364(6446), 1128-1130.

Rodríguez, A., Alfaro, E. J., \& Cortés, J. (2021). Spatial and temporal dynamics of the hydrology at Salinas Bay, Costa Rica, Eastern Tropical Pacific. Revista de Biología Tropical, 69(Supplement 2), S105-S126.

Samper-Villarreal, J., Bourg, A., Sibaja-Cordero, J. A., \& Cortés, J. (2014). Presence of a Halophila baillonii Asch. (Hydrocharitaceae) seagrass meadow and associated macrofauna on the Pacific coast of Costa Rica. Pacific Science, 68, 435-444. 
Samper-Villarreal, J., Cambronero-Bolaños, R., Heidemeyer, M., Mora-Vargas, M., \& Mora-Vargas, R. (2020). Characterization of seagrasses at two new locations in the Eastern Tropical Pacific (El Jobo and Matapalito, Costa Rica). Aquatic Botany, 165, 103237.

Samper-Villarreal, J., van Tussenbroek, B. I., \& Cortés, J. (2018). Seagrasses of Costa Rica: from the mighty Caribbean to the dynamic meadows of the Eastern Tropical Pacific. Revista de Biología Tropical, 66(Supplement 1), S53-S65.

Santagata, S. (2015). Phoronida. In A. Wanninger (Ed.), Evolutionary Developmental Biology of Invertebrates (Vol. 2 Lophotrochozoa (Spiralia), pp. 231-246). Wien: Springer.

Sibaja-Cordero, J. A., \& Cortés, J. (2008). Vertical zonation of rocky intertidal organisms in a seasonal upwelling area (Eastern Tropical Pacific). Revista de Biología Tropical, 56(Supplement 4), S91-S104.

Sibaja-Cordero, J. A., \& Vargas-Zamora, J. A. (2006) Zonación vertical de epifauna y algas en litorales rocosos del Golfo de Nicoya, Costa Rica. Revista de Biología Tropical, 54(Supplement 1), S49-S67.

Stuhldreier, I., Sánchez-Noguera, C., Rixen, T., Cortés, J., Morales, A., \& Wild, C. (2015). Effects of seasonal upwelling on inorganic and organic matter dynamics in the water column of eastern Pacific coral reefs. PLoS ONE, 10(11), e0142681.
Suárez, A., Riosmena-Rodríguez, R., \& Cortés, J. (2021). Checklist of invertebrates and conspicuous fishes in rocky reefs and Sargassum beds in the North Pacific of Costa Rica. Revista de Biología Tropical, 69(Supplement 2), S150-S193.

Tortolero-Langarica, J. J. A., Carricart-Ganivet, J. P., Cupul-Magaña, A. L., \& Rodríguez-Troncoso, A. P. (2017). Historical insights on growth rates of the reef-building corals Pavona gigantea and Porites panamensis from the Northeastern tropical Pacific. Marine Environmental Research, 132, 23-32.

Vargas-Castillo, R., \& Cortés, J. (2019). New records of marine decapods and stomatopods in Área de Conservación Guanacaste (ACG): four years of marine biodiversity inventorying. Marine Biodiversity Records, 12(1), 21.

Worm, B., Barbier, E. B., Beaumont, N., Duffy, J. E., Folke, C., Halpern, B. S., Jackson, J. B. C., Lotze, H. K., Micheli, F., Palumbi, S. R., Enric, S., Selkoe, K. A., Stachowicz, J. J., \& Watson, R. (2006). Impacts of biodiversity loss on ocean ecosystem services. Science, 314(5800), 787-790.

Zamora-Trejos, P., \& Cortés, J. (2009). Los manglares de Costa Rica: el Pacífico norte. Revista de Biología Tropical, 57(3), 473-488. 